\title{
Macular morphology and response to ranibizumab treatment in patients with wet age-related macular degeneration
}

This article was published in the following Dove Press journal:

Clinical Ophthalmology

15 June 2016

Number of times this article has been viewed

\section{Nikolaos Dervenis Saad Younis}

Western Eye Hospital, Imperial College Healthcare NHS Trust, London, UK
Correspondence: Nikolaos Dervenis Western Eye Hospital, Imperial College Healthcare NHS Trust, I53-I73 Marylebone Road, London NWI $5 \mathrm{QH}, \mathrm{UK}$

Tel +442033I26666

Email nikosdervenis@gmail.com
Purpose: The purpose of this study was to assess whether specific characteristics of spectral domain optical coherence tomography (SD-OCT) affect structural and functional outcomes and number of injections needed in ranibizumab $(0.05 \mathrm{~mL}$ of $10 \mathrm{mg} / \mathrm{mL}$ Lucentis solution)-treated wet age-related macular degeneration (AMD) patients.

Patients and methods: This retrospective case series included 62 newly diagnosed wet AMD patients treated with three monthly intravitreal ranibizumab injections followed by monthly follow-up and pro re nata retreatment. The presence of dome-shaped pigment epithelial detachment (PED), disruption of the retinal pigment epithelium (RPE), and subretinal and intraretinal fluid was associated with changes in Early Treatment of Diabetic Retinopathy Study visual acuity, central macular thickness (CMT), and number of injections needed during the 6-month follow-up.

Results: The presence of PED was associated with lower values of CMT at presentation $(399 \mu \mathrm{m}$ $[ \pm 132 \mu \mathrm{m}]$ vs $310 \mu \mathrm{m}[ \pm 51 \mu \mathrm{m}], P=0.005)$. The presence of RPE disruption was associated with worse visual acuity in month $6(0.36[ \pm 0.22]$ vs $0.61[0.45], P=0.027)$ and fewer injections (4.23 $[ \pm 0.92]$ vs $3.55[ \pm 0.60], P=0.007)$. The presence of intraretinal fluid at presentation was associated with worse visual acuity outcomes in month $4(P=0.045)$ but not in month 6 .

Conclusion: The dome-shaped PED was associated with lower CMT at presentation, but it did not affect response to treatment. RPE disruption was associated with worse functional outcomes with fewer injections. Intraretinal fluid at presentation may suggest delayed response to treatment. Individualized SD-OCT analysis could lead to individualized approach to wet AMD patients. SD-OCT can offer imaging biomarkers to assess the prognosis of anti-VEGF treatment in AMD patients.

Keywords: AMD, spectral domain optical coherence tomography, prognosis, anti-VEGF, ranibizumab

\section{Introduction}

The introduction of anti-VEGF treatment has improved the quality of life of wet age-related macular degeneration (AMD) patients. ${ }^{1}$ Moreover, modern imaging equipment, including spectral domain optical coherence tomography (SD-OCT), has enabled us to improve our understanding about the disease itself and the structural damage it causes to the macula. ${ }^{2}$ However, the classification scheme for wet AMD most widely used today is still based on fundus fluorescein angiography (FFA) findings. It was proposed $>20$ years ago by the Macular Photocoagulation Study Group that defined choroidal neovascular membrane (CNVM) types based on their location and pattern of hyperfluorescence (classic, occult, extra-, juxta-, subfoveal). ${ }^{3}$ This CNVM classification was useful in the 
pre anti-VEGF era as it associated FFA morphology with the treatment guidelines for wet AMD patients.

Gass $^{4}$ suggested that the location of neovascular complex with respect to the retinal pigment epithelium (RPE) might be relevant in predicting response to certain treatments and proposed an alternative classification of CNVM (type I: sub-RPE CNVM, type II: subretinal CNVM). Anti-VEGF could penetrate differently in different layers of the retina and the choroid, and therefore, CNVM location could affect treatment. Moreover, permanent functional damage of the retina could have developed even in cases where the structure improves after treatment. ${ }^{4}$

A new classification scheme based on modern imaging modalities (SD-OCT) should be associated with qualitative and quantitative factors identified in SD-OCT affecting response to anti-VEGF treatment.

The primary purpose of our study was to assess whether SD-OCT characteristics affect functional and structural outcomes in ranibizumab-treated wet AMD subjects. The secondary purpose of our study was to assess whether SD-OCT characteristics affect visual acuity (VA) at presentation and the number of injections needed in ranibizumab-treated wet $\mathrm{AMD}$ patients.

\section{Patients and methods}

This is a retrospective analysis of a series of patients being followed at the Medical Retina Clinic, Western Eye Hospital, London, UK. Ethical approval was obtained from the local hospital Ethics Committee prior to commencement of the study and all study procedures adhered to the Declaration of Helsinki. All participants provided informed consent for the retrospective data analysis we performed. The patients were followed monthly at the Medical Retina Clinic, and detailed ophthalmic examination was completed during every visit. This included Early Treatment of Diabetic Retinopathy Study VA measurement, applanation tonometry, and dilated fundus slit lamp examination. Demographic data and ophthalmic and medical history were also recorded during the first visit. The number of injections during the 6-month follow-up was also recorded for the purpose of this study. FFA was performed during the first visit of the patient to confirm the presence of CNVM secondary to AMD. Macula optical coherence tomography (OCT) was performed with the Cirrus SD-OCT (Carl Zeiss Meditec AG, Jena, Germany) using the $512 \times 128$ scan pattern, where a $6 \times 6 \mathrm{~mm}$ area of the retina is scanned with 128 horizontal lines, each consisting of 512 A scans per line. Only quality scores of at least 5 out of 10 points were used for this study.

The inclusion criteria for our study were first-treated wet AMD eyes in the Medical Retina Clinic of Western
Eye Hospital. The exclusion criteria included previous AMD treatment, scores $<5$ out of 10 in the quality score of the Cirrus OCT, polypoidal choroidal vasculopathy, other retinal pathology (for example, myopic CNVM), other ocular pathology affecting VA, history of ocular trauma, history of eye surgery during the last 6 months before AMD diagnosis, and follow-up $<6$ months after the AMD diagnosis.

The treatment protocol used was based on the PrONTO study, which suggests a pro re nata treatment following the initial loading dose of three intravitreal ranibizumab $(0.05 \mathrm{~mL}$ of $10 \mathrm{mg} / \mathrm{mL}$ Lucentis solution) injections. ${ }^{5}$

The analyzed OCT characteristics included the central macular thickness (CMT), the presence of dome-shaped pigment epithelial detachment (PED), the presence of an obvious RPE discontinuity, and the presence of subretinal or intraretinal fluid. The dome-shaped PED was defined as a domed space formed between the RPE and Bruch's membrane filled with mixed hyperreflective and hyporeflective materials. RPE discontinuity was defined as a hyporeflective gap in the hyperreflective line corresponding to the RPE in OCT. Figure 1 shows the presence of a dome-shaped PED, and Figure 2 shows the presence of RPE discontinuity and subretinal fluid. Figure 3 shows the presence of intraretinal fluid. A single grader assessed all the macula scans for the presence of the characteristics mentioned above. A reproducibility study was conducted before the beginning of the study, and it did not show significant variability in the grading outcomes. More specifically, ten randomly selected OCT scans from the AMD patients were graded twice ( 2 weeks apart) by the grader of the study, and the results were compared. The results showed high accordance for the grading.

Statistical analysis was performed using Statistical Package for the Social Sciences for Windows (Version

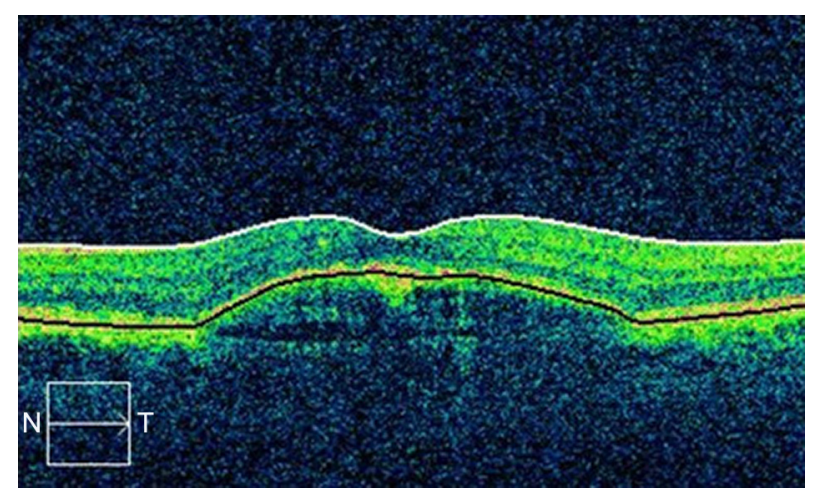

Figure I Dome-shaped PED.

Abbreviations: PED, pigment epithelial detachment; $\mathrm{N}$, nasal; $\mathrm{T}$, temporal. 


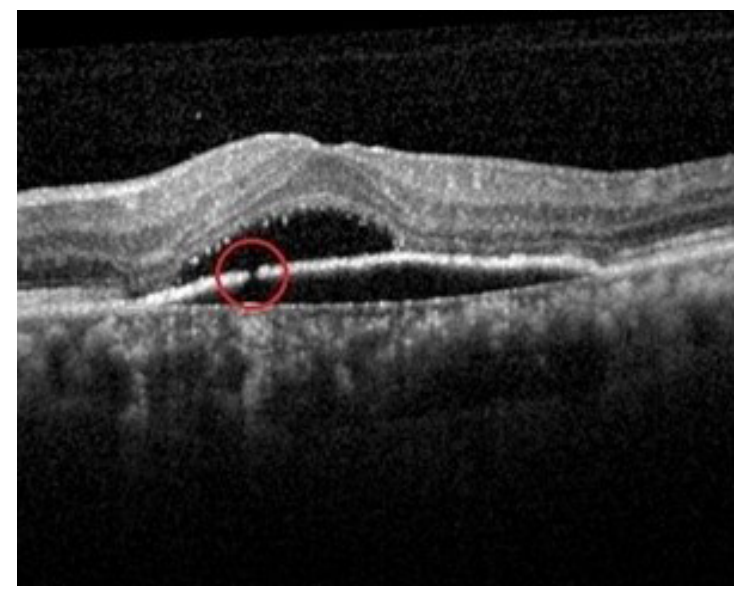

Figure 2 RPE discontinuity and subretinal fluid.

Note: Area of discontinuity shown in the red circle.

Abbreviation: RPE, retinal pigment epithelium.

18.0; SPSS Inc., Chicago, IL, USA). Independent samples $t$-test was used to compare the structural and functional outcomes and number of injections between the different groups. $P$-values $<0.05$ were considered statistically significant.

\section{Results}

A total of 62 eyes of 62 newly diagnosed wet AMD patients (29 males and 33 females) were included in the study. The mean age of our subjects was 72.9 years, and the mean Early Treatment of Diabetic Retinopathy Study VA at presentation was $0.59( \pm 0.27)$ improving to $0.49( \pm 0.38)$ in the fourth month of treatment. The mean number of injections performed in each eye during the 6 months of follow-up was $3.89( \pm 0.80)$. The patient characteristics are listed in Table 1.

In all, 28 of our patients had a dome-shaped PED, 34 had a disruption of the RPE, 42 had subretinal fluid, and 32 had

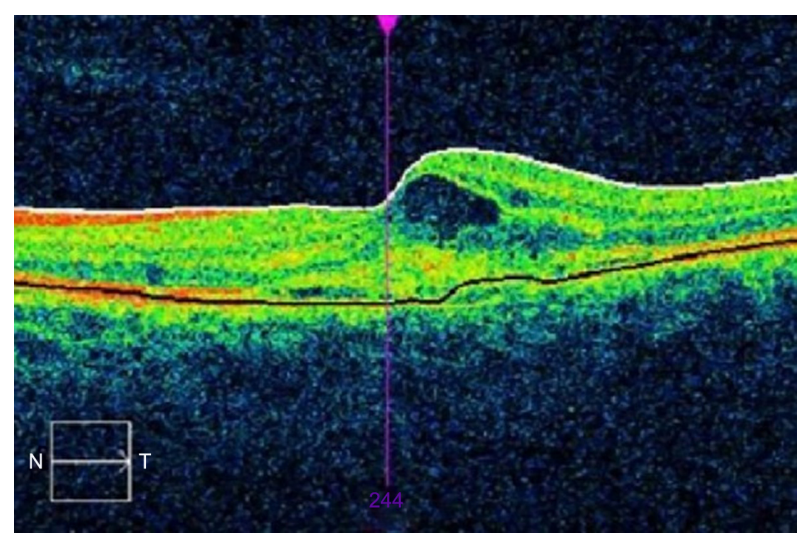

Figure 3 Intraretinal fluid. Abbreviations: $\mathrm{N}$, nasal; $\mathrm{T}$, temporal.
Table I Characteristics of our population

\begin{tabular}{ll}
\hline Variable, mean (SD) & $\mathbf{N}=\mathbf{6 2}$ \\
\hline Age (years) & $72.9(8.8)$ \\
Sex (male/female) & $29 / 33$ \\
VA at presentation & $0.59(0.27)$ \\
VA in month 4 & $0.49(0.38)$ \\
VA in month 6 & $0.51(0.38)$ \\
CMT at presentation $(\mu \mathrm{m})$ & $368(118.8)$ \\
CMT in month 4 $(\mu \mathrm{m})$ & $267(70.8)$ \\
CMT in month 6 $(\mu \mathrm{m})$ & $283(74.5)$ \\
Number of injections & $3.89(0.80)$ \\
\hline
\end{tabular}

Abbreviations: VA, visual acuity; CMT, central macular thickness; SD, standard deviation.

intraretinal fluid. The OCT characteristics are summarized in Table 2 .

Table 3 shows the functional and structural characteristics and the number of injections in PED eyes vs non-PED eyes. The PED eyes showed lower values of CMT thickness at presentation, but this did not affect the functional and structural characteristics and the number of injections during the course of treatment.

Table 4 summarizes the functional and structural characteristics and the number of injections in RPE discontinuity vs non-RPE discontinuity eyes. RPE discontinuity was associated with worse functional outcomes in month 6 with less injections. Further analysis of the SD-OCT scans showed that out of the 34 RPE discontinuity eyes, in 12 eyes, the discontinuity was located in the subfoveal area and in 22 eyes, the discontinuity was located in the juxta-foveal area.

Table 5 summarizes the functional and structural characteristics and the number of injections according to the presence of subretinal fluid. The presence of subretinal fluid did not affect VA outcomes, the CMT, or the number of injections in our case series.

Table 6 summarizes the functional and structural characteristics and number of injections according to the presence of intraretinal fluid. Intraretinal fluid was associated with worse functional outcomes in month 4 but not in month 6 .

Table 2 OCT characteristics in our population

\begin{tabular}{ll}
\hline OCT characteristic & n (\%) \\
\hline Dome-shaped PED, no (\%)/yes (\%) & $34(55.5) / 28(45.5)$ \\
RPE discontinuity, no (\%)/yes (\%) & $28(45.2) / 34(54.8)$ \\
Subretinal fluid, no (\%)/yes (\%) & $20(32.3) / 42(67.7)$ \\
Intraretinal fluid, no (\%)/yes (\%) & $30(48.4) / 32(51.6)$ \\
\hline
\end{tabular}

Abbreviations: OCT, optical coherence tomography; PED, pigment epithelial detachment; RPE, retinal pigment epithelium. 
Table 3 Functional and structural characteristics of dome-shaped PED vs non-PED eyes

\begin{tabular}{|c|c|c|}
\hline Variable & Mean (SD) & $P$-value \\
\hline \multicolumn{3}{|c|}{ VA at presentation } \\
\hline No PED & $0.56(0.25)$ & 0.50 \\
\hline PED & $0.62(0.31)$ & \\
\hline \multicolumn{3}{|c|}{ VA in month 4} \\
\hline No PED & $0.51(0.43)$ & 0.78 \\
\hline PED & $0.46(0.39)$ & \\
\hline \multicolumn{3}{|c|}{ VA in month 6} \\
\hline No PED & $0.55(0.46)$ & 0.50 \\
\hline PED & $0.47(0.36)$ & \\
\hline \multicolumn{3}{|c|}{ CMT at presentation } \\
\hline No PED & $399(132)$ & 0.005 \\
\hline PED & $310(5 \mathrm{I})$ & \\
\hline \multicolumn{3}{|c|}{ CMT in month 4} \\
\hline No PED & $27 \mid(77)$ & 0.50 \\
\hline PED & $255(6 I)$ & \\
\hline \multicolumn{3}{|c|}{ CMT in month 6} \\
\hline No PED & $279(77)$ & 0.55 \\
\hline PED & $293(75)$ & \\
\hline \multicolumn{3}{|c|}{ Number of injections } \\
\hline No PED & $3.85(0.88)$ & 0.86 \\
\hline PED & $3.81(0.74)$ & \\
\hline
\end{tabular}

Abbreviations: PED, pigment epithelial detachment; VA, visual acuity; CMT, central macular thickness; SD, standard deviation.

\section{Discussion}

The treatment of neovascular AMD has been revolutionized in the anti-VEGF era, and the prognosis of the disease has been greatly improved. Anti-VEGF therapies have been proven to reduce or even reverse visual loss

Table 4 Functional and structural characteristics and number of injections in RPE discontinuity vs non-RPE discontinuity eyes

\begin{tabular}{|c|c|c|}
\hline & Mean (SD) & $P$-value \\
\hline \multicolumn{3}{|l|}{ VA at presentation } \\
\hline No RPE discontinuity & $0.52(0.27)$ & 0.11 \\
\hline RPE discontinuity & $0.64(0.26)$ & \\
\hline \multicolumn{3}{|l|}{ VA in month 4} \\
\hline No RPE discontinuity & $0.41(0.32)$ & 0.22 \\
\hline RPE discontinuity & $0.54(0.48)$ & \\
\hline \multicolumn{3}{|l|}{ VA in month 6} \\
\hline No RPE discontinuity & $0.36(0.22)$ & 0.027 \\
\hline RPE discontinuity & $0.61(0.45)$ & \\
\hline \multicolumn{3}{|l|}{ CMT at presentation } \\
\hline No RPE discontinuity & $345(78)$ & 0.50 \\
\hline RPE discontinuity & $372(135)$ & \\
\hline \multicolumn{3}{|l|}{ CMT in month 4} \\
\hline No RPE discontinuity & $300(77)$ & 0.003 \\
\hline RPE discontinuity & $230(5 \mathrm{I})$ & \\
\hline \multicolumn{3}{|l|}{ CMT in month 6} \\
\hline No RPE discontinuity & $295(64)$ & 0.44 \\
\hline RPE discontinuity & $278(82)$ & \\
\hline \multicolumn{3}{|l|}{ Number of injections } \\
\hline No RPE discontinuity & $4.23(0.92)$ & 0.007 \\
\hline RPE discontinuity & $3.55(0.60)$ & \\
\hline
\end{tabular}

Abbreviations: RPE, retinal pigment epithelium; VA, visual acuity; CMT, central macular thickness; SD, standard deviation.
Table 5 Functional and structural characteristics and number of injections in subretinal fluid vs no subretinal fluid eyes

\begin{tabular}{|c|c|c|}
\hline & Mean (SD) & $P$-value \\
\hline \multicolumn{3}{|l|}{ VA at presentation } \\
\hline No subretinal fluid & $0.62(0.26)$ & 0.92 \\
\hline Subretinal fluid & $0.59(0.30)$ & \\
\hline \multicolumn{3}{|l|}{ VA in month 4} \\
\hline No subretinal fluid & $0.63(0.52)$ & 0.20 \\
\hline Subretinal fluid & $0.42(0.39)$ & \\
\hline \multicolumn{3}{|l|}{ VA in month 6} \\
\hline No subretinal fluid & $0.65(0.53)$ & 0.25 \\
\hline Subretinal fluid & $0.48(0.36)$ & \\
\hline \multicolumn{3}{|l|}{ CMT at presentation } \\
\hline No subretinal fluid & $399(112)$ & 0.22 \\
\hline Subretinal fluid & $348(115)$ & \\
\hline \multicolumn{3}{|l|}{ CMT in month 4} \\
\hline No subretinal fluid & $236(53)$ & 0.15 \\
\hline Subretinal fluid & $273(73)$ & \\
\hline \multicolumn{3}{|l|}{ CMT in month 6} \\
\hline No subretinal fluid & $273(92)$ & 0.47 \\
\hline Subretinal fluid & $29 \mid(70)$ & \\
\hline \multicolumn{3}{|l|}{ Number of injections } \\
\hline No subretinal fluid & $3.3(0.8 \mathrm{I})$ & 0.14 \\
\hline Subretinal fluid & $3.9(0.79)$ & \\
\hline
\end{tabular}

Abbreviations: VA, visual acuity; CMT, central macular thickness; SD, standard deviation.

in these patients. ${ }^{6}$ However, because of the variability in responses across different patients and because of the fluctuation in response of the same patient, the predictors for VA outcomes after anti-VEGF treatment have been investigated. ${ }^{7,8}$

Table 6 Functional and structural characteristics and number of injections in intraretinal fluid vs no intraretinal fluid eyes

\begin{tabular}{lll}
\hline & Mean (SD) & P-value \\
\hline VA at presentation & & \\
No intraretinal fluid & $0.54(0.22)$ & 0.22 \\
Intraretinal fluid & $0.63(0.30)$ & \\
VA in month 4 & & \\
$\quad$ No intraretinal fluid & $0.36(0.20)$ & 0.045 \\
Intraretinal fluid & $0.62(0.47)$ & \\
VA in month 6 & & \\
$\quad$ No intraretinal fluid & $0.44(0.29)$ & \\
Intraretinal fluid & $0.57(0.45)$ & \\
CMT at presentation & & \\
$\quad$ No intraretinal fluid & $360(133)$ & \\
Intraretinal fluid & $364(102)$ & 0.96 \\
CMT in month 4 & & \\
No intraretinal fluid & $279(78)$ & \\
Intraretinal fluid & $250(55)$ & \\
CMT in month 6 & & \\
No intraretinal fluid & $290(77)$ & \\
Intraretinal fluid & $280(77)$ & \\
Number of injections & & \\
No intraretinal fluid & $3.94(0.70)$ & \\
Intraretinal fluid & $3.73(0.90)$ &
\end{tabular}

Abbreviations: $\mathrm{VA}$, visual acuity; CMT, central macular thickness. 
Genetic, demographic, and clinical characteristics have been associated so far in the literature with the response to anti-VEGF treatment in wet AMD patients. ${ }^{9-11}$ SD-OCT characteristics have been proposed to serve as a new classification scheme for the disease as well. ${ }^{12}$ There are many obvious advantages with the use of SD-OCT characteristics to improve the accuracy of prognosis for AMD: it is quick, noninvasive, and part of the routine assessment of wet AMD subjects. ${ }^{13}$

Our study assessed whether baseline OCT characteristics can differentiate the functional and structural outcomes as well as the number of injections needed (therefore, the possibility of early recurrence or the speed of response to treatment) during the initial 6-month follow-up.

In our population, the presence of a dome-shaped PED was associated with lower values of CMT at presentation, but this did not have clinical implications regarding the response to treatment or the number of injections needed. The presence of fibrovascular PED is a well-established characteristic of wet AMD. ${ }^{13}$ The types of CNVM have also been classified based on their relationship with the RPE to sub-PRE (type I) or subretinal (type II). ${ }^{4}$ It seems from our results that the presence of a large lesion underneath the RPE is consistent with less pathological features over the RPE; therefore, CMT values are closer to normal.

The presence of an obvious RPE discontinuity in our population was associated with worse functional outcomes after 6 months of follow-up. RPE discontinuity is a characteristic that can be easily identified in SD-OCT images presenting as a hyporeflective gap in the hyperreflective RPE layer. Worse VA is actually achieved despite the fact that CMT is lower after 4 months of treatment in the RPE discontinuity group. The number of injections needed was also lower in this group, which could be explained either by the macula getting dry quicker (due to improved penetration of anti-VEGF to the sub-RPE space through the area of discontinuity) or by the VA dropping below the treatment threshold. RPE discontinuity could also signify photoreceptor disruption because of the proximity of photoreceptors to RPE and therefore permanent functional damage. To our knowledge, there are no previous studies assessing the role of RPE discontinuity in the outcomes of anti-VEGF therapy for wet AMD. It seems that the presence of defined RPE discontinuity is an adverse prognostic factor for anti-VEGF treatment response in our patients.

Although the presence of subretinal fluid was not associated with treatment outcomes in our case series, intraretinal fluid was associated with worse VA outcomes after the initial loading dose of four injections. However, VA outcomes after month 6, structural outcomes, and number of injections were not different between the groups. The presence of intraretinal fluid was associated with worse VA outcomes in the Comparison of Age-related Macular Degeneration Treatments Trials as well. ${ }^{14}$ It seems from our data that there is a delay in the response to the presence of intraretinal fluid at baseline; however, this did not affect treatment outcomes after 6 months in our study. Different studies conducted in different populations with different methodologies may give different findings for similar questions. Moreover, it was residual intraretinal fluid analyzed in the Comparison of Age-related Macular Degeneration Treatments Trials ${ }^{14}$ and not baseline intraretinal fluid.

There are some limitations with the design of our study. First of all, it is a small, nonrandomized, retrospective study with a limited follow-up time. The variables assessed were also subjective, but a reproducibility study was conducted before the beginning of the study that did not show significant variability. Other factors, including genetic or demographic variables, could also be assessed. However, it is one of the few studies associating macula structure with treatment outcomes in wet AMD subjects.

\section{Conclusion}

The presence of a dome-shaped PED was associated with lower values of CMT at presentation, but it did not affect treatment outcomes. The presence of an RPE discontinuity was associated with worse VA outcomes. The presence of intraretinal fluid did not affect treatment outcomes after 6 months; however, the response was delayed as VA was worse after the initial loading dose of three injections.

\section{Disclosure}

The authors report no conflicts of interest in this work.

\section{References}

1. Finger RP, Guymer RH, Gillies MC, Keeffe JE. The impact of antivascular endothelial growth factor treatment on quality of life in neovascular age-related macular degeneration. Ophthalmology. 2014;121(6): 1246-1251.

2. Sayanagi K, Sharma S, Yamamoto T, Kaiser PK. Comparison of spectral-domain versus time-domain optical coherence tomography in management of age-related macular degeneration with ranibizumab. Ophthalmology. 2009;116(5):947-955.

3. Group MPS. Argon laser photocoagulation for neovascular maculopathy: five-year results from randomized clinical trials. Arch Ophthalmol. 1991;109(8):1109.

4. Gass JDM. Biomicroscopic and histopathologic considerations regarding the feasibility of surgical excision of subfoveal neovascular membranes. Am J Ophthalmol. 1994;118(3):285-298.

5. Lalwani GA, Rosenfeld PJ, Fung AE, et al. A variable-dosing regimen with intravitreal ranibizumab for neovascular age-related macular degeneration: year 2 of the PrONTO Study. Am J Ophthalmol. 2009;148(1):43.e-58.e.

6. Lim LS, Mitchell P, Seddon JM, Holz FG, Wong TY. Age-related macular degeneration. Lancet. 2012;379(9827):1728-1738. 
7. Ying G-S, Maguire MG, Daniel E, et al; Comparison of Age-Related Macular Degeneration Treatments Trials (CATT) Research Group. Association of baseline characteristics and early vision response with 2-year vision outcomes in the comparison of AMD treatments trials (CATT). Ophthalmology. 2015;122(12):2523.e1-2531.e1.

8. Ying G-S, Huang J, Maguire MG, et al; Comparison of Age-related Macular Degeneration Treatments Trials Research Group. Baseline predictors for one-year visual outcomes with ranibizumab or bevacizumab for neovascular age-related macular degeneration. Ophthalmology. 2013;120(1):122-129.

9. Krishnan R, Arora R, De Salvo G, et al. Vitreomacular traction affects anti-vascular endothelial growth factor treatment outcomes for exudative age-related macular degeneration. Retina. 2015;35(9):1750-1756.

10. Brantley MA Jr, Fang AM, King JM, Tewari A, Kymes SM, Shiels A. Association of complement factor $\mathrm{H}$ and LOC387715 genotypes with response of exudative age-related macular degeneration to intravitreal bevacizumab. Ophthalmology. 2007;114(12):2168-2173.
11. Smailhodzic D, Muether PS, Chen J, et al. Cumulative effect of risk alleles in CFH, ARMS2, and VEGFA on the response to ranibizumab treatment in age-related macular degeneration. Ophthalmology. 2012; 119(11):2304-2311.

12. Freund KB, Zweifel SA, Engelbert M. Do we need a new classification for choroidal neovascularization in age-related macular degeneration? Retina. 2010;30(9):1333-1349.

13. Keane PA, Patel PJ, Liakopoulos S, Heussen FM, Sadda SR, Tufail A. Evaluation of age-related macular degeneration with optical coherence tomography. Surv Ophthalmol. 2012;57(5):389-414.

14. Jaffe GJ, Martin DF, Toth CA, et al. Macular morphology and visual acuity in the comparison of age-related macular degeneration treatments trials. Ophthalmology. 2013;120(9):1860-1870.
Clinical Ophthalmology

\section{Publish your work in this journal}

Clinical Ophthalmology is an international, peer-reviewed journal covering all subspecialties within ophthalmology. Key topics include: Optometry; Visual science; Pharmacology and drug therapy in eye diseases; Basic Sciences; Primary and Secondary eye care; Patient Safety and Quality of Care Improvements. This journal is indexed on

\section{Dovepress}

PubMed Central and CAS, and is the official journal of The Society of Clinical Ophthalmology (SCO). The manuscript management system is completely online and includes a very quick and fair peer-review system, which is all easy to use. Visit http://www.dovepress.com/ testimonials.php to read real quotes from published authors. 